\title{
High-Throughput Screening of Blood Donors for Twelve Human Platelet Antigen Systems Using Next-Generation Sequencing Reveals Detection of Rare Polymorphisms and Two Novel Protein-Changing Variants
}

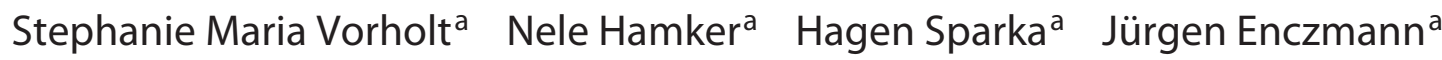 \\ Thomas Zeiler $^{\mathrm{b}}$ Tanja Reimer $^{\mathrm{b}}$ Johannes Fischer ${ }^{\mathrm{a}}$ Vera Balz ${ }^{\mathrm{a}}$ \\ a Institute for Transplantation Diagnostics and Cell Therapeutics, University Hospital Düsseldorf, Düsseldorf,

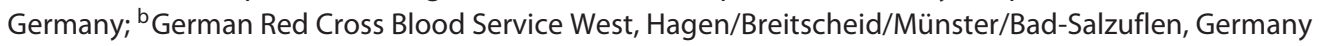

\section{Keywords \\ Human platelet antigens · Next-generation sequencing • High throughput}

\begin{abstract}
Background: Exposure to non-matching human platelet alloantigens (HPA) may result in alloimmunization. Antibodies to HPA can be responsible for post-transfusion purpura, refractoriness to donor platelets, and fetal and neonatal alloimmune thrombocytopenia. For the supply of compatible apheresis platelet concentrates, the HPA genotypes are determined in a routine manner. Methods: Here, we describe a novel method for genotyping twelve different HPA systems simultaneously, including HPA-1 to HPA-5, HPA-9w, HPA10w, HPA-16w, HPA-19w, HPA-27w, and the novel HPA-34w by means of amplicon-based next-generation sequencing (NGS). Blood donor samples of 757 individuals with a migration background and 547 of Western European ancestry were genotyped in a mass-screening setup. An in-house software was developed for fast and automatic analysis. TaqMan assay and Sanger sequencing results served for validation of the NGS workflow. Finally, blood donors were divided in several groups based on their country of origin and the
\end{abstract}

allele frequencies were compared. Results: For 1,299 of 1,304 samples (99.6\%) NGS was successfully performed. The concordance with TaqMan assay and Sanger sequencing results was $99.8 \%$. Allele-calling dropouts that were observed for two samples with the TaqMan assay caused by rare single nucleotide polymorphisms were resolved by NGS. Additionally, twenty rare and two novel variants in the coding regions of the genes ITGB3, GPB1A, ITGBA2, and CD109 were detected. The determined allele frequencies were similar to those published in the gnomAD database. Conclusions: No significant differences were observed in the distribution of allele frequencies of HPA- 1 through HPA- 5 and HPA-15 throughout the analyzed groups except for a lower allele frequency for the HPA-1b allele in the group of donors with Southern Asian ancestry. In contrast, other nucleotide variants that have not yet been phenotypically characterized occurred three times more often in blood donors with a migration background. High-throughput amplicon-based NGS is a reliable method for screening HPA genotypes in a large sample cohort simultaneously. It is easily upgradeable for genotyping additional targets without changing the setup or the analysis pipeline. Mass-screening methods will help building up blood donor registries to provide matched blood products. 


\section{Introduction}

The provision of optimally matched blood products is a challenge in countries of Western Europe with regard to the high number of citizens with a background of migration. The BluStar.NRW project was initiated to analyze the distribution of blood group phenotypes in this population. Further, it aims to enlarge the number of blood donors who are negative for high-prevalence antigens such as Vel or both of the Duffy antigens Fy(a) and Fy(b). On the other hand, blood donors who are positive for low-prevalence antigens that can cause alloimmunization in antigen-negative patients can be identified, too.

Exposure to the human platelet antigens (HPA) also may elicit immune response in individuals lacking the exposed antigen. Immunization to these antigens due to pregnancy or transfusion can cause severe clinical disorders such as fetal and neonatal alloimmune thrombocytopenia (FNAIT), posttransfusion purpura, and platelet transfusion refractoriness to random donor platelets [1]. It is supposed that FNAIT is caused by HPA antibodies only [2]. Thrombocytopenia in FNAIT can be severe, with intracranial hemorrhage occurring in $10 \%$ to $30 \%$ of severe FNAIT cases. Several types of neonatal treatment have been proposed, of which transfusion of HPA-compatible platelets is most effective [3]. For efficient therapy with compatible platelet components, not only a register of HPA-typed blood donors is essential [4] but also a fast and reliable HPA typing method.

Currently, 35 different HPA alloantigens are known. All of them are results from amino acid replacements giving rise to various epitopes in the extracellular domains of the glycoproteins GPIa, GPIba, GPIIb, GPIIIa, and CD109 as published by the IPD-HPA database [5] (www. ebi.ac.uk/ipd/). These proteins play an important role in platelet adhesion and aggregation by serving as receptors for specific ligands [4].

Among the 35 HPA antigens defined by immune sera, the six bi-allelic HPA systems HPA-1 through HPA-5 and HPA- 15 are clinically most relevant and alloantibodies against both of the allelic variants have been observed. For the remaining 23 antigens, alloantibodies have been described against the thetical but not the antithetical antigen [1].

In the recent years, several methods have been developed for the detection of the most important HPA epitopes. Amongst them are restriction fragment length polymorphism analysis, allele-specific oligonucleotide hybridization, polymerase chain reaction using allelespecific primers (PCR-SSP), and real-time PCR protocols using either melting-curve analysis or the TaqMan technology as reviewed in Veldhuisen et al. [6]. Those HPA typing methods are restricted to detect the known polymorphisms, only. More recently, next-generation se- quencing (NGS) methods have gained attention in genotyping blood group antigens as well as platelet antigens [7-10]. Besides previously described polymorphisms, also novel variations can be identified by using NGS methods.

With respect to HPA, Davey et al. [7] used a set of 47 samples to establish an NGS approach for genotyping of the entire set of 29 HPA systems. Here, a custom exome capture system was developed and results of the NGS approach were compared to previously obtained TaqMan assay or SSP genotyping results. In this setup, 21 samples were pooled in one particular NGS run. They succeeded to genotype 46 out of 47 samples.

Orzińska et al. [9] developed an amplicon-based approach which served for genotyping of a set of blood groups (RHCE, RHD, FY, JK, and MNS) along with HPA1 to HPA- 5 and HPA- 15 in a cohort of 57 samples. Here, 14 different fragments were amplified for each sample and 48 samples were pooled into one NGS experiment.

Both studies were performed as preliminary studies. They show the feasibility to use NGS platforms for reliable genotyping of HPA alloantigens. However, the number of samples that can be analyzed simultaneously are relatively low.

Therefore, the goal of our study was the development and validation of an amplicon-based NGS test system for HPA alloantigens for large-scale blood donor typing in order to provide HPA-antigen-negative platelet products to highly alloimmunized patients. This included the integration of an in-house developed software for the analysis of the known polymorphisms and the detection of novel variants to allow a fast and reliable genotyping workflow. As a proof of principle, we investigated the most common HPA alloantigens HPA-1 through HPA-3, HPA-5, and HPA- 15 as well as the rare variations of HPA-4, HPA- $9 \mathrm{w}$, HPA-10w, HPA-16w, HPA-19w, and HPA-27w in a cohort of 1,304 samples of different ethnic ancestries. In addition, a recently described novel HPA antigen, HPA$34 \mathrm{w}$, which is currently not approved by the Platelet Nomenclature Committee [1], was included [11]. In our approach, up to 760 samples were analyzed in one single NGS experiment. In addition, we describe the distribution of allele frequencies of HPAs in groups of people from different areas of the world.

\section{Material and Methods}

\section{Sample Cohort}

As part of the BluStar.NRW project, 757 blood donors with a migration background were recruited for blood donation by the German Red Cross Blood Service. We further enrolled 547 blood samples from a random cohort of blood donors of mainly Western European ancestry from the Institute for Hemotherapy and Transfusion Medicine at the University Hospital Düsseldorf, Germany.
Vorholt/Hamker/Sparka/Enczmann/ Zeiler/Reimer/Fischer/Balz 
Table 1. Summary of molecular of HPA systems in the present study

\begin{tabular}{|c|c|c|c|c|c|c|c|c|c|}
\hline \multirow[t]{2}{*}{ HPA type } & \multirow{2}{*}{$\begin{array}{l}\text { Glycopro- } \\
\text { tein }\end{array}$} & \multirow{2}{*}{$\begin{array}{l}\text { Gene } \\
\text { locus }\end{array}$} & \multirow{2}{*}{$\begin{array}{l}\text { Accession } \\
\text { number }\end{array}$} & \multirow{2}{*}{$\begin{array}{l}\text { Chro- } \\
\text { mosome }\end{array}$} & \multirow{2}{*}{ SNP ID } & \multirow[t]{2}{*}{ Allele name } & \multirow[t]{2}{*}{ Nucleotide } & \multicolumn{2}{|c|}{ Amino acid } \\
\hline & & & & & & & & precursor & $\begin{array}{l}\text { mature } \\
\text { protein }\end{array}$ \\
\hline HPA-1a & GPIIIa & ITGB3 & NM_000212 & 17 & rs5918 & ITGB3*001 & c.176T & Leu 59 & Leu 33 \\
\hline HPA-1b & GPIIIa & ITGB3 & NM_000212 & 17 & rs5918 & ITGB3*002 & c. $176 \mathrm{C}$ & Pro 59 & Pro 33 \\
\hline HPA-2a & GPIBa & $G P 1 B A$ & NM_000173 & 17 & rs 6065 & $\mathrm{GP} 1 \mathrm{BA} * 001$ & c. $482 \mathrm{C}$ & Thr 161 & Thr 145 \\
\hline HPA-2b & GPIBa & $G P 1 B A$ & NM_000173 & 17 & rs6065 & GP1BA*002 & c. $482 \mathrm{~T}$ & Met 161 & Met 145 \\
\hline HPA-3a & GPIIB & $I T G A 2 B$ & NM_000419 & 17 & rs5911 & ITGA2B*001 & c. $2621 \mathrm{~T}$ & Iso 874 & Iso 843 \\
\hline HPA-3b & GPIIB & $I T G A 2 B$ & NM_000419 & 17 & rs5911 & ITGA2B*002 & c. $2621 \mathrm{G}$ & Ser 874 & Ser 843 \\
\hline HPA-4a & GPIIIa & ITGB3 & NM_000212 & 17 & rs5917 & ITGB3*001 & c. $506 \mathrm{G}$ & Arg 169 & Arg 143 \\
\hline HPA-4b & GPIIIa & ITGB3 & NM_000212 & 17 & rs5917 & ITGB3*005 & c. $506 \mathrm{~A}$ & Gln 169 & Gln 143 \\
\hline HPA-5a & GPIa & ITGA2 & NM_002203 & 5 & rs10471371 & ITGA $2 * 001$ & c. $1600 \mathrm{G}$ & Glu 534 & Glu 505 \\
\hline HPA-5b & GPIa & ITGA2 & NM_002203 & 5 & rs10471371 & ITGA $2 * 002$ & c. $1600 \mathrm{~A}$ & Lys 534 & Lys 505 \\
\hline \multirow[t]{2}{*}{ HPA-9bw } & GPIIB & ITGA2B & NM_000419 & 17 & rs74988902 & ITGA2B*003 & c. $2621 \mathrm{G}$ & Ser 847 & Ser 843 \\
\hline & & & & & & & c. $2602 \mathrm{~A}$ & Met 868 & Met 837 \\
\hline HPA-10bw & GPIIIa & ITGB3 & NM_000212 & 17 & rs 200358667 & ITGB3*003 & c. $263 \mathrm{~A}$ & Gln 88 & $G \ln 62$ \\
\hline HPA-15a & CD109 & CD109 & NM_133493 & 6 & rs10455097 & $\mathrm{CD} 109^{*} 001$ & c. $2108 \mathrm{C}$ & Ser 703 & Ser 682 \\
\hline HPA-15b & CD109 & CD109 & NM_133493 & 6 & rs10455097 & CD109*002 & c. $2108 \mathrm{~A}$ & Tyr 703 & Tyr 682 \\
\hline HPA-16bw & GPIIIa & ITGB3 & NM_000212 & 17 & & ITGB3*004 & c. $497 \mathrm{~T}$ & Iso 166 & Iso 140 \\
\hline HPA-19bw & GPIIIa & ITGB3 & NM_000212 & 17 & rs80115510 & & c. $487 \mathrm{C}$ & Gln 163 & $\mathrm{G} \ln 137$ \\
\hline HPA-27bw & GPIIB & ITGA2B & NM_000419 & 17 & rs149468422 & & c. $2614 \mathrm{~A}$ & Met 872 & Met 841 \\
\hline HPA-34bw & GPIIIa & ITGB3 & NM_000212 & 17 & rs77774846 & & c.349T & Trp 117 & $\operatorname{Trp} 91$ \\
\hline
\end{tabular}

Source: IPD - the Immuno Polymorphism Database [5] except HPA-34w; Bertrand et al. [11] for HPA-34bw. No SNP-ID was assigned to HPA-16bw. HPA-19bw, HPA-27bw, and HPA-34bw are not enumerated with allele names, yet.

For method validation and single nucleotide polymorphism (SNP) analysis, all 1,304 samples were pooled leading to a group of investigation with a maximum genetic diversity. Donors declared their ethnic ancestry in a voluntary manner. Samples were grouped according to their country of origin in order to determine the respective allele frequencies. All blood donors gave written consent for the use of blood samples for research purposes. The use of blood samples for research use was approved by the ethics committee of University Hospital Düsseldorf.

\section{DNA Isolation}

Buffy coat samples were diluted with PBS in an equal ratio for further use. Genomic DNA was extracted by means of DNAQiamp ${ }^{\circledR} 96$ DNA Blood kit (Qiagen, Hilden, Germany) according to the manufacturer's instructions. At variance with the instructions, a prolonged incubation time of $30 \mathrm{~min}$ was used for lysis.

\section{HPA Systems under Investigation}

The five common HPA systems HPA-1, HPA-2, HPA-3, HPA5, and HPA-15 were investigated using a TaqMan assay as well as the NGS test. The rare HPA-4 system was equally analyzed using both assays. In addition, the rare HPA systems HPA-9w, HPA10w, HPA-16w, HPA-19w, and HPA-27w as well as the novel HPA-34w were present in the same amplicons of the genes ITGB3 and ITGA2B. Therefore, they were also included in the NGS test system. A summary of the molecular genetics of the HPA systems is given in Table 1.

\section{TaqMan Assay}

In order to validate the NGS test system, all samples were genotyped for HPA-1 through HPA-5 and HPA-15 using a TaqMan real-time assay which is used for HPA diagnostic in our laboratory in a routine manner. Amplification primers and probes are given in Table 2 [12-14]

Briefly, the real-time PCR assays were conducted in a total volume of $10 \mu \mathrm{L}$ containing 15-30 ng genomic DNA, $5 \mu \mathrm{L}$ Master Mix with the passive reference dye ROX (Biozym Blue Probe qPCR Mix Separate ROX, Hamburg, Germany), and primers and allele-discriminating probes in a final concentration of $0.4 \mu \mathrm{M}$ each. Amplification primers and probes were purchased from Eurofins Operon (Ebersberg, Germany). All reactions were performed using identical conditions. First, genomic DNA was denaturated at $95^{\circ} \mathrm{C}$ for $3 \mathrm{~min}$. The following 35 amplification cycles consisted of two consecutive steps: denaturation at $95^{\circ} \mathrm{C}$ for $15 \mathrm{~s}$ and annealing and elongation at $60^{\circ} \mathrm{C}$ for $30 \mathrm{~s}$. Data analysis was done with StepOne ${ }^{\mathrm{TM}}$ software v2.3 and TaqMan Genotyper software (Thermo Fisher Scientific, Germany).

\section{Next-Generation Sequencing}

Primer Design and Testing

An amplicon-based approach was used to analyze the common HPA systems HPA-1 through HPA-3, HPA-5, and HPA-15. PCR primers were designed with the help of Primer3 [15] by using reference sequences as published in the IPD-HPA database (https:// www.ebi.ac.uk/ipd/hpa/). The primers were checked for additional SNPs using the SNPCheck software (https://genetools.org/SNPCheck/snpcheck.htm) which may impair binding to the template and, as a consequence, result in allele dropout. Primers were purchased from Biolegio (Nijmegen, The Netherlands). In order to ensure specificity, all primers were tested in single PCR amplification protocols. The amplified fragments were subsequently analyzed by Sanger sequencing. An overview of the amplification fragments, primers, and amplicon size of covered HPA alloantigens is given in Table 3. 


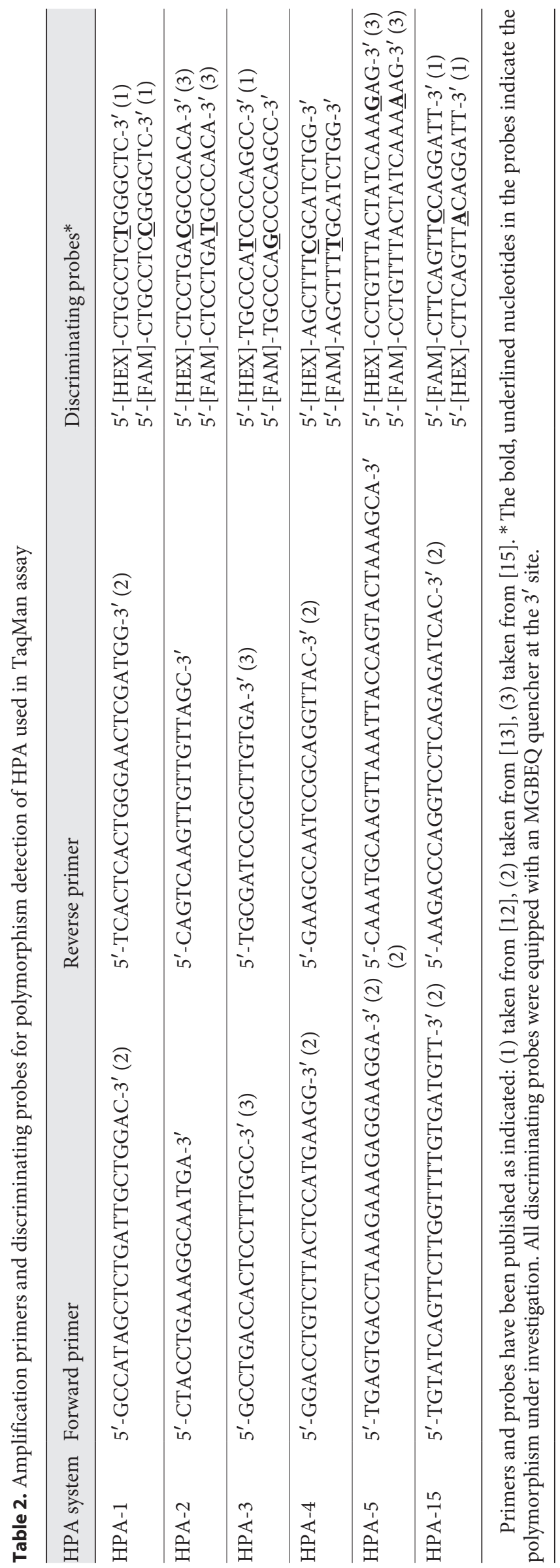

Library Preparation and Sequencing

For the NGS test, the six fragments were amplified in a single multiplex PCR reaction. For each sample, 50-100 ng genomic DNA, 7.5 $\mu$ L KAPA2G Fast Multiplex Mix (Kapa Biosystems, Cape Town, South Africa), and $1.5 \mu \mathrm{L}$ of each primer with a final concentration of $1 \mu \mathrm{M}$ were combined in a total volume of $15 \mu \mathrm{L}$. PCR reactions were performed using the following conditions: $95^{\circ} \mathrm{C}-$ 3 min; 30 cycles of $95^{\circ} \mathrm{C}-15 \mathrm{~s}, 63^{\circ} \mathrm{C}-45 \mathrm{~s}, 72^{\circ} \mathrm{C}-45 \mathrm{~s} ; 72^{\circ} \mathrm{C}-$ $3 \mathrm{~min}$.

After a cleanup step using paramagnetic beads, amplification products underwent a second-round PCR that added sample-specific barcodes and Illumina-compatible adapter sequences. Two different sample barcodes at the $5^{\prime}$-prime and the $3^{\prime}$-prime, respectively, were chosen for each sample resulting in dual-labelled amplicons. Using different combinations of $5^{\prime}$-prime and $3^{\prime}$-prime barcodes enabled the analysis of more than the usually pooled 96 samples per NGS experiment.

The barcoding PCR was carried out using the following conditions: $3 \mu \mathrm{L}$ purified PCR product, $5 \mu \mathrm{L}$ KAPA HiFi HotStart ReadyMix (Kapa Biosystems, Cape Town, South Africa), $2 \mu \mathrm{L}$ barcoding primer mix $(0.4 \mu \mathrm{M}$, each $) ; 95^{\circ} \mathrm{C}-5 \mathrm{~min} ; 15$ cycles $95^{\circ} \mathrm{C}-20 \mathrm{~s}, 65^{\circ} \mathrm{C}-15 \mathrm{~s}, 72^{\circ} \mathrm{C}-20 \mathrm{~s} ; 72^{\circ} \mathrm{C}-1 \mathrm{~min}$.

Amplification products of 760 samples and 552 samples, respectively, were pooled and underwent a second cleanup step. Afterwards, libraries were quantified by real-time PCR. Here, primers were directed to the tails of PCR fragments which are identical to the Illumina adapter sequences. Cloned PCR amplificates of three different fragments served as quantification standards covering the range of fragment length and GC percentage of fragments that are included in the NGS test system. After quantification, $6 \mathrm{pM}$ of the resulting library was used for a $2 \times 272$ cycle run on a MiSeq instrument (Illumina Inc., San Diego, CA, USA) using a Standard v3 cartridge according to the manufacturer's instructions.

The entire library preparation workflow was performed in 384well plate to obtain a high-throughput assay. It was conducted with the help of automatic pipetting systems (Biomek 4000 and Biomek $\mathrm{NX}^{\mathrm{P}}$, Beckman Coulter, Krefeld, Germany).

For verification of repeatability, seven samples were included in every NGS test. The depth of coverage, quality of reads, and genotyping results were compared.

\section{Evaluation}

Runs that were included in this study had to pass the following criteria: cluster density between 800 and $1,500 \mathrm{k} / \mathrm{mm}^{2}$, more than $70 \%$ of clusters pass the filter, Q30 score must be more than $70 \%$.

Data Analysis

The analysis of the read sequences was performed by an inhouse software (Blood Group_Analyser) approach. The sequence data generated by the MiSeq instrument were analyzed by applying the following steps: Primary data analysis was performed using the MiSeq Reporter software to generate a pair of fastq-files for each sample. The paired-end reads were assembled using PANDAseq [16] generating the raw sequence data. For each of these sequences, the locus and region to which the sequence belongs to were determined by the use of a decision tree. To remove sequencing errors which were introduced during PCR amplification or by the MiSeq instrument, an error correction step was performed taking all raw sequences belonging to the same locus and region into account. Next, allele groups were generated by comparing the error-corrected sequences with a library of reference sequences for each allele. Here, all alleles whose sequence matches the corrected read sequence in the relevant locus and region are determined. 
Table 3. Amplification primers used in NGS test

\begin{tabular}{|c|c|c|c|c|c|}
\hline Fragment & Gene & Forward primer & Reverse primer & $\begin{array}{l}\text { Amplicon } \\
\text { size }\end{array}$ & $\begin{array}{l}\text { Targeted } \\
\text { HPA system }\end{array}$ \\
\hline 1 & ITGB3 & 5'-AGCTATTGGGAAGTGGTAGGGC-3' & 5'-TCCATTCTCTTGCCСАСАССТ-3' & $436 \mathrm{bp}$ & $\begin{array}{l}\text { HPA-1, } \\
\text { HPA-10w, } \\
\text { HPA-34w }\end{array}$ \\
\hline 2 & ITGB3 & 5'-AGTCCCAACTGTATCCAAATCTGCT-3' & 5'-ATGCTGTCCTGGCGTCTGG-3' & $427 \mathrm{bp}$ & $\begin{array}{l}\text { HPA-4, } \\
\text { HPA-16w, } \\
\text { HPA-19w }\end{array}$ \\
\hline 3 & $G P 1 B A$ & $\begin{array}{l}5^{\prime} \text {-TCCCACAATCAGCTGCAAAGC-3' } \\
5^{\prime} \text {-TCCCACAATCAGCTACAAAGCC-3' } \\
5^{\prime} \text {-TCCCACAATCAGCTCCAA-3' }\end{array}$ & 5'-TCACACTGCACACTGGCCAC-3' & $494 \mathrm{bp}$ & HPA-2 \\
\hline 4 & ITGA2B & $\begin{array}{l}\text { 5'-CCTGACCACTCCTTTGCCCC-3' } \\
5^{\prime} \text {-GCCTGACCACTCCTTTGCCC-3' }\end{array}$ & 5'-TTTCCACGGGCTTGCTCACA-3' & $471 \mathrm{bp}$ & $\begin{array}{l}\text { HPA-3, } \\
\text { HPA-9w, } \\
\text { HPA-27w }\end{array}$ \\
\hline 5 & ITGA2 & $\begin{array}{l}\text { 5'-AGACGTGCTCTTGGTAGGTG-3' } \\
\text { 5'-AGACGTGCTGTTGGTAGGTG-3' }^{\prime} \text { 5'-CAGATGTGCTCTTGGTAGGTG-3' }^{\prime} \\
5^{\prime} \text {-CAGACATGCTCTTGGTAGGTG-3' }\end{array}$ & 5'-GACCTCTCATGGAAAATGGCAGT-3' & $313 \mathrm{bp}$ & HPA-5 \\
\hline 6 & CD109 & 5'-TCAGTTCTTGGTTTTGTGATGTT-3' & 5'-TCTTCTGGCCTTTGGAGATGGT-3' & $434 \mathrm{bp}$ & HPA-15 \\
\hline
\end{tabular}

Table 4. Summary of results obtained by NGS and TaqMan assays for 1,304 donors

\begin{tabular}{|c|c|c|c|c|c|c|}
\hline & HPA-1 & HPA-2 & HPA-3 & HPA-4 & HPA-5 & HPA-15 \\
\hline Total number of analyzed alleles & 2,608 & 2,608 & 2,608 & 2,608 & 2,608 & 2,608 \\
\hline Number of failed samples (NGS) & 4 & 5 & 4 & 4 & 4 & 5 \\
\hline Number of failed analyses (TaqMan) & - & - & 5 & 3 & 3 & 3 \\
\hline Number of compared samples & 1,300 & 1,299 & 1,295 & 1,297 & 1,297 & 1,296 \\
\hline Number of discrepant results & 1 & - & - & - & - & 1 \\
\hline Number of false NGS results & - & - & - & - & - & - \\
\hline Number of false TaqMan results & 1 & - & - & - & - & 1 \\
\hline
\end{tabular}

In case of the glycoprotein GPIIIa, where two fragments are amplified to cover the HPA systems HPA-1, HPA-4, HPA-10w, HPA-16w, HPA-19w, and HPA-34w, intersections of all allele groups of both regions were calculated and the particular combination of intersections which matched the sample's data best was determined.

\section{Statistical Analysis}

Blood donors with a migration background were subdivided into eight groups. Allele frequencies of each of the groups were determined by direct counting. The validity of Hardy-Weinberg equilibrium was tested by calculating expected numbers of subjects for each genotype using $2 \times$ af $[\mathrm{a}] \times$ af $[\mathrm{b}] \times n$ for heterozygotes and af $[\mathrm{a} \text { or } \mathrm{b}]^{2} \times n$ for homozygotes, where af $[\mathrm{a}]$ and af $[\mathrm{b}]$ are the allele frequencies of the a- and b-alleles of HPA-1 to HPA-3, HPA5 , and HPA-15, respectively, and $n$ is the number of subjects typed. Subsequently, the values were compared to the observed numbers. Goodness-of-fit between the observed (Obs) and expected (Exp) values was tested using the following formula: $\chi^{2}=\operatorname{sum}(\mathrm{Obs}-$ Exp $)^{2} /$ Exp, for 1 degree of freedom. $p$ values $\leq 0.01$ were considered statistical significant [17].

\section{Results}

Next-Generation Sequencing as a Reliable Genotyping Method for HPA

A novel amplicon-based NGS approach was developed for genotyping twelve biallelic HPA systems simultaneously in a high-throughput manner. A well-established TaqMan assay which is used for HPA alloantigen diagnostic in our laboratory in a routine manner served for validation of the NGS genotyping method for six of the HPA systems (HPA-1 through HPA-5 and HPA-15).

1,299 of 1,304 samples (99.6\%) were successfully genotyped for all twelve HPA systems using the NGS approach, including the common HPA-1 through HPA-3, HPA-5, and HPA-15 alloantigens as well as the rare systems HPA4, HPA-9w, HPA-10w, HPA-16w, HPA-19w, and HPA$27 \mathrm{w}$. The remaining five samples failed for parts or the entire set of HPA alloantigens for technical reasons, e.g., insufficient amounts of DNA input or problems with the cleanup step of this particular sample (Table 4). 
Table 5. Allele frequencies of HPA-1 through HPA-5 and HPA-15 in different ethnic groups

\begin{tabular}{|c|c|c|c|c|c|c|c|c|c|c|c|c|c|}
\hline \multirow[t]{2}{*}{ Population } & \multirow{2}{*}{$\begin{array}{l}\text { Samples, } \\
n\end{array}$} & \multicolumn{2}{|c|}{ HPA-1 } & \multicolumn{2}{|c|}{ HPA-2 } & \multicolumn{2}{|c|}{ HPA-3 } & \multicolumn{2}{|c|}{ HPA-4 } & \multicolumn{2}{|c|}{ HPA-5 } & \multicolumn{2}{|c|}{ HPA-15 } \\
\hline & & $\mathrm{a}$ & $\mathrm{b}$ & a & b & a & $\mathrm{b}$ & $\mathrm{a}$ & $\mathrm{b}$ & $\mathrm{a}$ & $\mathrm{b}$ & a & b \\
\hline WEUR & 547 & 0.839 & 0.161 & 0.912 & 0.088 & 0.579 & 0.419 & 1 & 0 & 0.895 & 0.105 & 0.498 & 0.502 \\
\hline ARAB & 120 & 0.913 & 0.088 & 0.875 & 0.125 & 0.650 & 0.350 & 1 & 0 & 0.854 & 0.146 & 0.500 & 0.400 \\
\hline TURK & 166 & 0.877 & 0.123 & 0.886 & 0.114 & 0.642 & 0.358 & 1 & 0 & 0.852 & 0.148 & 0.503 & 0.497 \\
\hline EECCA & 83 & 0.886 & 0.114 & 0.867 & 0.133 & 0.657 & 0.343 & 1 & 0 & 0.873 & 0.127 & 0.398 & 0.602 \\
\hline IRAN & 35 & 0.857 & 0.143 & 0.871 & 0.129 & 0.543 & 0.429 & 0.948 & 0.014 & 0.914 & 0.086 & 0.443 & 0.557 \\
\hline SSAF & 11 & 0.938 & 0.063 & 1 & 0 & 0.594 & 0.406 & 1 & 0 & 0.938 & 0.063 & 0.594 & 0.406 \\
\hline SAS & 18 & 0.972 & 0.028 & 0.917 & 0.083 & 0.694 & 0.306 & 1 & 0 & 0.889 & 0.111 & 0.556 & 0.444 \\
\hline SEEA & 18 & 0.906 & 0.094 & 1 & 0 & 0.656 & 0.344 & 1 & 0 & 0.938 & 0.063 & 0.635 & 0.375 \\
\hline OTH & 306 & 0.866 & 0.134 & 0.864 & 0.136 & 0.574 & 0.423 & 1 & 0 & 0.894 & 0.106 & 0.484 & 0.516 \\
\hline
\end{tabular}

WEUR, West Europe; ARAB, Eritrea, Libya, Egypt, Morocco, Algeria, Tunisia, Lebanon, Iraq, Saudi-Arabia, Jordan, and Syria; TURK, Turkey; EECCA, East Europe, Caucasus, Central Asia: Greece, Macedonia, Bosnia and Herzegovina, Romania, Bulgaria, Croatia, Albania, Serbia, Kosovo, Russia, Armenia, Georgia, Azerbaijan, Afghanistan, Kazakhstan, and Kyrgyzstan; IRAN, Iran; SSAF, sub-Saharan Africa: South Africa, Togo, Nigeria, Ghana, Burkina Faso, and Kenia; SAS, South Asia: India, Pakistan, Bangladesh, and Sri Lanka; SEEA, Southeast/East Asia: China, Thailand, Vietnam, Nepal, Singapore, Malaysia, South Korea, and Japan; OTH, other migrants: country of origin unknown.

For the five common HPA systems and the rare HPA4 alloantigen, amplicon-based NGS genotyping results were in accordance to previously obtained TaqMan assay data for 1,295 samples (99.8\%). The majority of the detected alleles corresponded to the common a- and b-alleles of these HPA systems.

For the rare HPA systems HPA-9w, HPA-10w, HPA16w, HPA-19w, HPA-27w, and HPA-34w, specificity was validated by Sanger sequencing. Here, the low frequency variants HPA-10bw, HPA-16bw, HPA-19bw, HPA$27 \mathrm{bw}$, and HPA-34bw were not present in the set of samples. The HPA-9bw allele was found in heterozygosity in 5 of 1,299 samples $(0.004 \%)$ and the HPA- $4 \mathrm{~b}$ allele in heterozygosity in one sample $(0.0008 \%)$.

\section{Data Quality and Allele Balance}

The depth of coverage for each of the fragments was well above the minimum of 50 reads per amplicon, with 114-1,168 (mean 465), 144-1,198 (mean 602), 150-1,570 (mean 678), 317-1,813 (mean 1,099), 237-2,066 (mean 781), and 134-1,451 (mean 449) for fragments covering HPA-1, HPA-2, HPA-3, HPA-4, HPA-5, and HPA-15, respectively.

Seven samples which were analyzed in both NGS runs show similar depth of coverage and quality scores. The genotyping results obtained from both runs were identical for this set of samples.

For all HPA systems under investigation but HPA-2, the depth of coverage of each allele obtained for heterozygous samples ranged nearby the expected $50-50 \%$ distribution $(46.7-53.7 \%, 48.4-56.5 \%, 47.9-56.2 \%$, and $40.6-55.3 \%$ for the a-alleles of HPA- $1,-3,-5$, and -15 , respectively) indicating that PCR amplification is not bi- ased. One sample was found to carry a HPA- $4 \mathrm{~b}$ allele. Here, the coverage for the a-allele was $48 \%$ and the coverage for the b-allele was $52 \%$. In case of HPA-9w, three heterozygous donors were present. The coverage of the HPA-9bw allele was $48.3,47.2$, and $44.0 \%$. In contrast, the HPA-2 system showed a divergent pattern with a slightly higher coverage of HPA-2a in heterozygous samples (54.2-69.4\% for HPA-2a). Sanger sequencing analysis was performed of the primer binding sites for donor samples which showed the highest coverage difference of HPA-2a and HPA-2b. We did not find any SNP in the contemplable region.

\section{Allele Distribution in Different Ethnic Groups}

The samples were divided into eight groups of different ethnic backgrounds. The genotyping results were used to analyze the allele frequencies of the HPA systems HPA- 1 through HPA-5 and HPA-15 (Table 5). The country of origin was known for 451 blood donors with a migration background. Of these, 166 individuals were of Turkish ancestry. The remaining donors were collected in groups of Arabs, East Europeans including individuals from the Caucasus and Central Asia, the Iran, South Asia, Southeast and East Asia, and individuals from Sub-Saharan Africa (120, 83, 35, 18, 18, and 11 samples, respectively). Donors with unknown country of origin were grouped into "other migrants" (306 samples).

The most frequent genotypes were the homozygous aallele for the systems HPA-1, HPA-2, and HPA-5, whereas the heterozygous genotype was more present in HPA3 and HPA- 15 systems regardless of the population. We did not observe any significant differences in the distribution of allele frequencies of HPA- 1 through HPA- 5 and 
Table 6. Rare and novel polymorphisms in genes of the HPA system

\begin{tabular}{|c|c|c|c|c|c|c|c|c|c|c|}
\hline \multirow{2}{*}{$\begin{array}{l}\text { Glyco- } \\
\text { protein }\end{array}$} & \multirow{2}{*}{$\begin{array}{l}\text { Most } \\
\text { homologous } \\
\text { allele }\end{array}$} & \multirow[t]{2}{*}{ Location } & \multirow{2}{*}{$\begin{array}{l}\text { Nucleotide } \\
\text { change }^{\mathrm{a}}\end{array}$} & \multirow{2}{*}{$\begin{array}{l}\text { Codon } \\
\text { position }\end{array}$} & \multirow{2}{*}{$\begin{array}{l}\text { Type of } \\
\text { mutation }\end{array}$} & \multicolumn{2}{|c|}{ Amino acid change } & \multicolumn{2}{|c|}{ Allele frequency } & \multirow[t]{2}{*}{ SNP ID } \\
\hline & & & & & & precursor & $\begin{array}{l}\text { mature } \\
\text { protein }\end{array}$ & $\begin{array}{l}\text { gnomAD } \\
\text { database }^{b}\end{array}$ & $\begin{array}{l}\text { present } \\
\text { study }\end{array}$ & \\
\hline $\mathrm{GPIb}_{\alpha}$ & GP1BA*002 & Exon 2 & c. $655 \mathrm{C}>\mathrm{T}$ & 1 & missense & H219Y & H203Y & - & $7.621 \mathrm{e}-4$ & Novel \\
\hline GPIIIa & ITGB $3 * 001$ & Exon 4 & c. $517 \mathrm{~A}>\mathrm{G}$ & 1 & missense & S173G & S147G & - & $7.621 \mathrm{e}-4$ & Novel \\
\hline GPIIIa & ITGB $3 * 001$ & Exon 3 & c. $180 \mathrm{C}>\mathrm{T}$ & 3 & synonymous & - & - & $1.698 \mathrm{e}-4$ & $1.524 \mathrm{e}-3$ & rs373101628 \\
\hline GPIIIa & ITGB3*002 & Exon 3 & c.197T >G & 2 & missense & L66R & L40R & $1.765 e-3$ & $2.231 \mathrm{e}-3$ & rs36080296 \\
\hline GPIIIa & ITGB3*002 & Exon 3 & c. $285 \mathrm{C}>\mathrm{T}$ & 3 & synonymous & - & - & $3.148 \mathrm{e}-3$ & $2.231 \mathrm{e}-3$ & rs151121691 \\
\hline GPIIIa & ITGB3*001 & Exon 3 & c. $342 \mathrm{~T}>\mathrm{C}$ & 3 & synonymous & - & - & $5.745 e-3$ & $2.231 \mathrm{e}-3$ & rs5920 \\
\hline GPIIIa & ITGB3*001 & Exon 4 & c. $368 \mathrm{C}>\mathrm{T}$ & 2 & missense & S123L & S97L & $1.988 \mathrm{e}-5$ & $7.621 \mathrm{e}-4$ & rs755704884 \\
\hline GPIIIa & ITGB3*001 & Exon 4 & c. $537 \mathrm{C}>\mathrm{T}$ & 3 & synonymous & - & - & $1.273 \mathrm{e}-4$ & $7.621 \mathrm{e}-4$ & rs770144031 \\
\hline GPIIIa & ITGB3*001 & Exon 4 & c. $575 \mathrm{~A}>\mathrm{G}$ & 2 & missense & Y192C & Y166C & $7.956 \mathrm{e}-7$ & $7.621 \mathrm{e}-4$ & rs777168504 \\
\hline $\mathrm{GPIb}_{\alpha}$ & GP1BA*001 & Exon 2 & c. $358 \mathrm{G}>\mathrm{A}$ & 1 & missense & V120I & V104I & $1.204 \mathrm{e}-5$ & $7.621 \mathrm{e}-4$ & rs780652188 \\
\hline $\mathrm{GPIb}_{\alpha}$ & GP1BA*001 & Exon 2 & c. $367 \mathrm{G}>\mathrm{A}$ & 1 & missense & V123I & V107I & $3.186 e-6$ & $1.524 \mathrm{e}-3$ & rs760616671 \\
\hline $\mathrm{GPIb}_{\alpha}$ & GP1BA*001 & Exon 2 & c. $390 \mathrm{G}>\mathrm{A}$ & 3 & synonymous & - & - & $9.229 \mathrm{e}-6$ & $7.621 \mathrm{e}-4$ & rs181416431 \\
\hline $\mathrm{GPIb}_{\alpha}$ & GP1BA*001 & Exon 2 & c. $409 \mathrm{C}>\mathrm{A}$ & 1 & missense & $\mathrm{R} 137 \mathrm{~S}$ & R121S & $4.013 e-7$ & $7.621 \mathrm{e}-4$ & rs1191148388 \\
\hline $\mathrm{GPIb}_{\alpha}$ & GP1BA*001 & Exon 2 & c. $461 \mathrm{C}>\mathrm{A}$ & 2 & missense & $\mathrm{T} 154 \mathrm{~N}$ & $\mathrm{~T} 138 \mathrm{~N}$ & $1.204 \mathrm{e}-6$ & $7.621 \mathrm{e}-4$ & rs1229994069 \\
\hline $\operatorname{GPIb}_{\alpha}$ & GP1BA*001 & Exon 2 & c. $483 \mathrm{G}>\mathrm{A}$ & 3 & synonymous & - & - & $1.601 \mathrm{e}-3$ & $1.524 \mathrm{e}-3$ & rs2243094 \\
\hline $\mathrm{GPIb}_{\alpha}$ & GP1BA*001 & Exon 2 & c. $634 \mathrm{C}>\mathrm{T}$ & 1 & missense & $\mathrm{L} 212 \mathrm{~F}$ & L196F & $4.012 \mathrm{e}-7$ & $7.621 \mathrm{e}-4$ & rs374770057 \\
\hline $\mathrm{GPIb}_{\alpha}$ & GP1BA*001 & Exon 2 & c.638dupCCT & 2 & duplication & L213dup & L197dup & $7.130 \mathrm{e}-6$ & $7.621 \mathrm{e}-4$ & rs749267498 \\
\hline $\operatorname{GPIb}_{\alpha}$ & GP1BA*001 & Exon 2 & c. $756 \mathrm{C}>\mathrm{T}$ & 3 & synonymous & - & - & $2.006 \mathrm{e}-5$ & $7.621 \mathrm{e}-4$ & rs745330132 \\
\hline $\mathrm{GPIb}_{\alpha}$ & GP1BA*001 & Exon 2 & c. $774 \mathrm{C}>\mathrm{T}$ & 3 & synonymous & - & - & & 0.0346 & \\
\hline $\mathrm{GPIb}_{\alpha}$ & GP1BA*002 & Exon 2 & c. $774 \mathrm{C}>\mathrm{T}$ & 3 & synonymous & - & - & 0.0216 & $2.231 \mathrm{e}-3$ & \\
\hline GPIIb & ITGA2B*001 & Exon 26 & c. $2725 \mathrm{G}>\mathrm{C}$ & 1 & missense & V909L & V878L & $7.105 e-5$ & $7.621 \mathrm{e}-4$ & rs573974230 \\
\hline CD109 & CD109*002 & Exon 19 & c. $2139 A>G$ & 3 & synonymous & - & - & $2.017 \mathrm{e}-4$ & $1.524 \mathrm{e}-3$ & rs 150533440 \\
\hline CD109 & CD109*001 & Exon 19 & c. $2194 \mathrm{G}>\mathrm{C}$ & 1 & missense & G732R & G711R & $1.912 \mathrm{e}-4$ & $7.621 \mathrm{e}-4$ & rs200492695 \\
\hline CD109 & CD109*002 & Exon 19 & c. $2213 \mathrm{C}>\mathrm{T}$ & 2 & missense & T738I & G717R & $3.811 \mathrm{e}-3$ & $3.049 \mathrm{e}-3$ & rs 138465270 \\
\hline
\end{tabular}

${ }^{a}$ Position in cDNA. ${ }^{b}$ gnomAD database available at: gnomad.broadinstitute.org [18]. ${ }^{c}$ This polymorphism was present with HPA-2a and HPA-2b. Nucleotide change: nucleotide numbers are given in relation to the reference sequence in the NCBI database. SNP, single nucleotide polymorphism.

Table 7. Rare and novel polymorphisms in introns of ITGB3 and ITGB2B

\begin{tabular}{|c|c|c|c|c|c|c|}
\hline \multirow[t]{2}{*}{ Gene } & \multirow{2}{*}{$\begin{array}{l}\text { Most homologous } \\
\text { allele }\end{array}$} & \multirow[t]{2}{*}{ Location } & \multirow{2}{*}{$\begin{array}{l}\text { Nucleotide } \\
\text { change }^{\mathrm{a}}\end{array}$} & \multicolumn{2}{|c|}{ Allele frequency } & \multirow[t]{2}{*}{ SNP ID } \\
\hline & & & & $\begin{array}{l}\text { gnomAD } \\
\text { database }^{b}\end{array}$ & $\begin{array}{l}\text { present } \\
\text { study }\end{array}$ & \\
\hline ITGB3 & ITGB3*001 & Intron 3 & c. $362-43 \mathrm{G}>\mathrm{T}$ & - & $7.621 \mathrm{e}-4$ & Novel \\
\hline ITGB3 & ITGB3*001 & Intron 2 & c. $166-48 \mathrm{C}>\mathrm{T}$ & $6.204 \mathrm{e}-4$ & $7.621 \mathrm{e}-4$ & rs184965646 \\
\hline ITGB3 & ITGB $3^{*} 001$ & Intron 2 & c. $166-40 \mathrm{C}>\mathrm{T}$ & 0.04712 & 0.08695 & rs988684 \\
\hline ITGB3 & ITGB3*001 & Intron 3 & c. $362-30 \mathrm{G}>\mathrm{A}$ & $8.900 \mathrm{e}-3$ & 0.02372 & rs147055245 \\
\hline ITGB3 & ITGB3*002 & Intron 4 & c. $614+15 \mathrm{C}>\mathrm{T}$ & $7.98 \mathrm{e}-6$ & $7.621 \mathrm{e}-4$ & rs376456408 \\
\hline ITGA2B & ITGA2B*001 & Intron 26 & c. $2727+14 C>G$ & $1.201 \mathrm{e}-5$ & $7.621 \mathrm{e}-4$ & rs573974230 \\
\hline
\end{tabular}

${ }^{a}$ Position in cDNA. ${ }^{b}$ gnomAD database available at: gnomad.broadinstitute.org [18]. Nucleotide change: nucleotide numbers are given in relation to the reference sequence in the NCBI database. SNP, single nucleotide polymorphism.

HPA-15 throughout the analyzed groups, except a lower allele frequency for the HPA-1b allele in the group of South Asia comprising individuals from India, Pakistan, Bangladesh, and Sri Lanka. The sample number in this group was too small to provide reliable statistical analysis. Genotype frequencies of HPA-1 to HPA-5 and HPA-15 were in accordance with the Hardy-Weinberg equilibrium for all populations.

With regard to the rare HPA systems, the b-allele of HPA-4 was found in one sample of a blood donor from Iran. Five samples carried the HPA-9bw allele. Of these, two alleles were detected in samples with Western European ancestry and one each in an Iranian and a Bulgarian blood donor. For the fifth sample, the ancestry has not been documented. The allele frequencies and the ethnic ancestries both fit well in population data as published by the 1,000 Genome and Genome Aggregation database (gnomAD) [18]. In addition, additional nucleotide variants which are not phenotypically characterized by now occurred three times more often in blood donors with a migration background (30/757 [0.04\%] versus 8/547 [0.015\%]; Tables 6 and 7). 
Divergent Results in NGS and TaqMan Assay

In two cases, the genotyping results differ between NGS test and TaqMan analysis (Table 4). In detail, one sample was found to be HPA-1ab in NGS but HPA-1aa in TaqMan assay. Here, the NGS revealed the presence of a silent nucleotide variation $(\mathrm{c} .180 \mathrm{C}>\mathrm{T})$ in the HPA-1a allele. This variation resides in the discriminating probe which was used in the TaqMan assay leading to a dropout of allele calling. The same variant was present in a second sample. Since this particular sample was genotyped HPA1aa using the NGS test, we did not observe an allele calling dropout in the TaqMan assay.

For the other sample an allele calling dropout was observed in HPA-15 in the TaqMan assay. Whereas the sample was genotyped HPA-15ab using the NGS test, the TaqMan assay result was HPA-15aa. In this case, an SNP (c.2194G>C, rs200492695) was found in the HPA-15b allele which is located in the binding region of the reverse primer of the TaqMan assay. A reduced binding capacity of the reverse primer due to a mismatch leads to a missing amplification of the respective allele and therefore to an incorrect interpretation of the genotype.

Additionally, two samples were found with an SNP (c.483G $>$ A) in the HPA-2a allele being directly adjacent to the SNP discriminating between the $a$ - and $b$-allele of HPA-2 in the gene GP1BA. Consequently, this SNP is located in the binding region of the discriminating probe for the TaqMan assay. Applying the NGS test, both samples were found to be homozygous for HPA-2a. Thus, the dropout of allele calling of this rare allele did not result in an incorrect genotyping in the TaqMan assay but probably would in case of heterozygosity.

\section{Additional Rare and Novel Nucleotide Variants}

Twenty-two rare, previously published nucleotide variants in the coding regions of the HPA genes were detected in 36 samples $(0.028 \%)$. Seventeen of those variants result in an altered protein but are not designated as an HPA system, yet. One additional variant (c.774C>T) was found to be coupled with both the a-allele and the b-allele of the HPA- 2 system ( 0.037 and $0.005 \%$, respectively). In addition, two novel amino acid changing polymorphisms were identified (Table 4). All SNPs were confirmed by means of Sanger sequencing.

The two variants are located on chromosome 17 . The first is located in gene GP1BA exon 2 and is characterized by a C-to-T transition at position c.655. This transition leads to an exchange from histidine to tryptophan at amino acid 203 of the mature glycoprotein $\mathrm{GPIb}_{\alpha}$. Exon 2 of GP1BA covers the HPA antigen HPA-2.

The second polymorphism shows an A-to-G exchange at position c.517 in gene ITGB3 exon 4 resulting in a replacement of serine to glycine at amino acid 147 on the mature GPIIIa protein. This exchange is only four amino acids apart from the HPA-4 a/b antigen determining position. Both amino acid exchanges are located on the extracellular part of the protein potentially resulting in novel antigens.

Further, six noncoding variants were identified. Although they are not likely to have impact on the phenotype, the nucleotide variation may reside at primer binding positions that are used for molecular diagnostic methods such as SSP, LightCycler, or TaqMan assays. Therefore, they are also included in the present study. Five of these variants have been described previously. For the gene ITGB3, one novel intron polymorphisms was found which is located upstream of exon 4 (Table 7).

\section{Discussion}

In this study, we present a novel NGS workflow that enables genotyping of twelve HPA alloantigen systems in a high-throughput manner. 1,304 randomly selected samples from different ethnic groups were used for establishing the workflow. Genotyping results were validated by TaqMan assay and/or Sanger sequencing.

Davey et al. [7] established an NGS protocol for the analysis of 29 HPA alloantigens. A target-enrichment protocol applying a customized HaloPlexHS Panel was used to analyze 46 samples. Up to 21 samples were pooled in one single NGS experiment at the same time. According to the current available index combinations for the target enrichment workflow, this method allows a maximum capacity of 96 samples to be sequenced in a single experiment.

In contrast, for the current NGS pipeline, we chose an amplicon-based pipeline with a two-step PCR protocol. The use of different combinations of $5^{\prime}$-prime and $3^{\prime}$-prime barcodes resulting in dual-indexed amplicons enables the analysis of up to 760 blood donor samples simultaneously. The entire workflow is fully automatable including data analysis by a customized in-house software created for the analysis of HPA alloantigens. The enlarged number of samples and automatic data analysis will provide donor registries with fast and reliable genotyping results. As the present workflow is based on a twostep PCR protocol only, material costs of no more than EUR 3 make it a fast and inexpensive technique.

$99.6 \%(1,299$ of 1,304$)$ samples were successfully genotyped for the entire set of 18 different HPA alloantigens using the NGS test. The repeated analysis of seven randomly chosen samples with similar depth of coverage and quality of reads and identical genotyping results reflects the repeatability and reliability of this novel method. The sample cohort covered a variety of different ethnic backgrounds. In the majority of cases, results obtained by our NGS method were identical to the results derived from TaqMan assays or Sanger sequencing method.
Vorholt/Hamker/Sparka/Enczmann/ Zeiler/Reimer/Fischer/Balz 
In total, we found two non-matching genotype results under all data from the 1,299 blood donor samples $(0.002 \%)$. Similar to our observations, Flegel et al. [10] and Boccoz et al. [8] reported genotyping discrepancies of $0.004 \%$ and $0.17-1 \%$, respectively, when comparing NGS data of different blood group panels to BeadChip, TaqMan assay, or RBC serological phenotype data. Both samples were found to have an allele calling dropout in the TaqMan assay due to different polymorphisms either located in the discriminating probe or in one of the amplification primers. Rare and novel polymorphisms at or in the near vicinity of the antigen-discriminating polymorphism have been described already for HPA-1, HPA-3, and HPA-5, making it necessary to include additional discriminating probes in the TaqMan assay design or novel primers in SSP analysis $[19,20]$.

In our sample cohort, we observed a synonymous mutation on the gene ITGB3 with the nucleotide exchange $\mathrm{C}>\mathrm{T}$ at position c.180 (rs373101628), which was reported earlier with an allele frequency of $0.0002 \%$. The SNP is only four bases downstream from the HPA-1a/b determining SNP and is coupled to the ITGB $3 * 001$ allele which encodes the HPA-1a alloantigen. The variant was found in two individuals with a migration background but the countries of origin for these particular samples were not documented. This rare allele was mainly seen for Caucasian (Non-Finnish) and East Asian people as reported in the gnom AD database [18].

Allele dropouts caused by variants located in the amplification primers may also be causative for miscalling of genotypes by molecular genetic methods. This is demonstrated by an allele dropout of HPA-15b in the TaqMan assay provoked by the SNP rs200492695 (c.2194G>C). This SNP was reported in the gnomAD database with an allele frequency of $1.912 \mathrm{e}-4$. Since this SNP is rare, it is often not taken into account when creating primers for genotyping assays such as SSP, TaqMan, or Luminex analysis.

In addition, one polymorphism (c.483G > A, rs2243094) which affects the binding of the discriminating probe for the HPA-2a/b alloantigen was found for two unrelated blood donors, both of them have migration background (one individual from Syria, one sample with unknown country of origin). This finding corresponds well with data from the gnomAD database where this polymorphism is found to have an allele frequency of 0.01705 in people of African ancestry, whereas it is rarely found in people with European (Non-Finnish) origin (allele frequency: $2.339 \mathrm{e}-5$ ). The detection of divergent results between different analysis methods emphasizes that primers and probes have to be chosen prudently and checked for SNPs taking the ethnic background of the analyzed samples into account.

Genotyping of 12 HPA Systems in a Mass Screening Amplicon-Based NGS Approach
Among all samples, none of the rare alleles were detected for the HPA systems HPA-10w, HPA-16w, HPA19w, HPA-27w, and HPA-34w. The balanced depth of coverage for more frequent alleles as well as for alleles carrying rare variants in heterozygous samples suggests that those alleles would have been detected. With regard to the HPA systems HPA-4 and HPA-9bw, the b-allele of HPA-4 was found in one sample of a blood donor from the Iran. Five samples carried the HPA-9bw allele. Of these, two alleles were detected in samples of Western European ancestry and one sample each of Bulgarian and Iranian origin. For the fifth sample, the country of origin was not documented. Both the allele frequencies and the ethnic ancestries fit well in population data as published by the gnomAD database.

Recently, two novel HPA alloantigens which provoke FNAIT were described [11, 21]. The alloantigens were designated as HPA-34bw and HPA-35bw by the authors. They are not approved by the Platelet Nomenclature Committee, yet [1]. One of these novel alloantigens, HPA-34bw, resides within the amplicon that was designed for the analysis of HPA-1 and HPA-10w. However, this particular nucleotide change was not observed in the investigated sample cohort. Likewise, two additional allelic variants of the HPA systems, HPA-1 and HPA-5, expanding these systems from biallelic to triallelic systems, were not found in the present study $[19,20]$.

In the cohort of 1,304 blood and platelet donors, twenty-two previously published and two novel variants were identified in the coding regions of the four genes ITGB3, $G P B 1 A, I T G A 2 B$, and CD109, whereas no additional polymorphism beside the a/b-SNP was found in the gene ITGA2. Fourteen of the identified variants result in an altered glycoprotein. All of the altered protein structures are exposed on the platelet surface, potentially giving rise to novel alloantigens. Two rare variants were found in blood donors of Western European ancestry only $\left(\mathrm{GP} 1 \mathrm{BA} * 001+\mathrm{c} .634 \mathrm{C}>\mathrm{T}\right.$ and $\mathrm{GP} 1 \mathrm{BA}{ }^{*} 001+$ c.638dupCCT), and four variants were detected in Western Europeans as well as donors with a migration background $\left(\mathrm{GP}_{1 \mathrm{BA}} * 001+\mathrm{c} .774 \mathrm{C}>\mathrm{T}, \mathrm{GP} 1 \mathrm{BA} * 00+\right.$ c. $774 \mathrm{C}>\mathrm{T}$, ITGB3*001 + c.197T $>\mathrm{G}$, and CD109*002+ c. $2213 \mathrm{C}>\mathrm{T}$ ). The remaining eighteen variants were exclusively found in blood donors with a migration background, reflecting the enhanced genetic diversity of these populations.

The genotype and allele frequencies among the analyzed ethnic cohorts do not differ significantly. The only exception is a lower frequency for HPA-1b in the group consisting of blood donors of Southern Asian ancestry (0.028). However, the sample number was too small to provide reliable statistical analysis. Bhatti et al. [22] investigated the frequencies of HPA- 1 to HPA- 5 and HPA-15 for different ethnic groups throughout Pakistan. Here, 
the frequency of HPA- $1 \mathrm{~b}$ ranged from 0.04 to 0.18 , with the lowest frequencies observed for Sindhis and the highest frequencies found for the group of Brahuis.

A variety of studies dealing with different populations and different sets of targeted HPA systems and analysis methods described similar allele frequencies in individuals originating from countries throughout the world, as summarized in Neffati et al. [23]. The allele frequencies for the other ethnic groups as well as for the remaining HPA systems did not differ from the reported data.

Amongst the molecules present on the cell membrane, not only HPAs but also human leukocyte antigens (HLA) play a significant role in alloimmunization in the setting of platelet transfusion. In addition, antibodies evoked by incompatibility to CD36 [24] or blood group antigens such as $\mathrm{A}$ and $\mathrm{B}$ of the $\mathrm{ABO}$ blood group have been observed [25]. HLA genotyping in organ or bone marrow transplantations is routinely performed. Likewise, in our laboratory, we developed an amplicon-based NGS workflow for genotyping the HLA genes $H L A-A,-B,-C$, $-D R B 1$, $-D R B 3,-D R B 4,-D R B 5,-D Q B 1$, and $-D P B 1$ in a highthroughput manner. Up to now, this workflow has served to genotype more than 75,000 stem cell donors which are registered at the German Stem Cell Donor Registry Düsseldorf [26]. For HLA genotyping, we analyze up to 380 samples simultaneously. The well-established HLA genotyping workflow and the novel HPA NGS workflow are similar with regard to the use of a two-step PCR protocol, dual primer barcoding which is used for sample indexing, run specifications, and the in-house developed analysis software. Hence, genotyping for HLA and HPA alloanti- gens may be combined in one NGS experiment. In addition, further targets, for example blood group antigens or human neutral antigens, can be easily included without changing the pipeline. The use of NGS makes mass screening for the establishment of antigen profiles feasible. This enables blood donor registries to identify rare donors in order to provide antigen-negative units given to alloimmunized patients and to improve their clinical outcomes.

\section{Acknowledgement}

We are grateful to Gabriele Tillmann, Gabriele Häger and Beata Maruhn-Debowski who helped with laboratory assistance.

\section{Statement of Ethics}

Blood donors gave written consent for the use of blood samples for research purposes. The use of blood samples for research was approved by the ethics committee of University Hospital Düsseldorf.

\section{Disclosure Statement}

The authors declare no conflict of interest.

\section{Funding Sources}

This study was funded by the European Regional Development Fund 2014-2020 (EFRE), Grant/Award Number: EFRE-0800961.

\section{References}

1 Metcalfe P, Watkins NA, Ouwehand WH, Kaplan C, Newman P, Kekomaki R, et al. Nomenclature of human platelet antigens. Vox Sang. 2003 Oct;85(3):240-5.

2 Taaning E. HLA antibodies and fetomaternal alloimmune thrombocytopenia: myth or meaningful? Transfus Med Rev. 2000 Jul; 14(3):275-80.

3 Serrarens-Janssen VM, Semmekrot BA, Novotny VM, Porcelijn L, Lotgering FK, Delemarre FM, et al. Fetal/neonatal allo-immune thrombocytopenia (FNAIT): past, present, and future. Obstet Gynecol Surv. 2008 Apr;63(4):239-52.

4 Rozman P. Platelet antigens. The role of human platelet alloantigens (HPA) in blood transfusion and transplantation. Transpl Immunol. 2002 Aug;10(2-3):165-81.

5 Robinson J, Halliwell JA, McWilliam H, Lopez R, Marsh SG. IPD-the Immuno Polymorphism Database. Nucleic Acids Res. 2013 Jan;41(Database issue):D1234-40.

6 Veldhuisen B, Porcelijn L, Ellen van der Schoot C, de Haas M. Molecular typing of human platelet and neutrophil antigens (HPA and HNA). Transfus Apheresis Sci. 2014 Apr; 50(2):189-99.
7 Davey S, Navarrete C, Brown C. Simultaneous human platelet antigen genotyping and detection of novel single nucleotide polymorphisms by targeted next-generation sequencing. Transfusion. 2017 Jun;57(6):1497-504.

8 Boccoz SA, Fouret J, Roche M, Lachuer J, Legras-Lachuer C, Corgier BP, et al. Massively parallel and multiplex blood group genotyping using next-generation-sequencing. Clin Biochem. 2018 Sep;60:71-6.

9 Orzińska A, Guz K, Mikula M, Kulecka M, Kluska A, Balabas A, et al. A preliminary evaluation of next-generation sequencing as a screening tool for targeted genotyping of erythrocyte and platelet antigens in blood donors. Blood Transfus. 2018 May;16(3):285-92.

10 Flegel WA, Gottschall JL, Denomme GA. Implementing mass-scale red cell genotyping at a blood center. Transfusion. 2015 Nov;55(11): 2610-5.

11 Bertrand G, Danger Y, Laurichesse M, Verite F, Renac V. A case of neonatal thrombocytopenia caused by maternal alloimmunization against a new platelet antigen (Bzha, HPA34bw) located on GPIIIa. Transfusion. 2019 Apr;59(4):1402-3.
12 Hansen CU. Human platelet antigen (HPA) 1-5 and 15 typing in an Egyptian population using sequence specific primer PCR and TaqMan technology including studies of four discrepancies in the HPA 2 system. Tromsø, Norway: University of Tromsø; 2006.

13 Ruan L, Pei B, Li Q. Multicolor real-time polymerase chain reaction genotyping of six human platelet antigens using displacing probes. Transfusion. 2007 Sep;47(9):163742.

14 Ficko T, Galvani V, Rupreht R, Dovc T, Rozman P. Real-time PCR genotyping of human platelet alloantigens HPA-1, HPA-2, HPA-3 and HPA-5 is superior to the standard PCRSSP method. Transfus Med. 2004 Dec;14(6): 425-32.

15 Untergasser A, Cutcutache I, Koressaar T, Ye J, Faircloth BC, Remm M, et al. Primer3-new capabilities and interfaces. Nucleic Acids Res. 2012 Aug;40(15):e115.

16 Masella AP, Bartram AK, Truszkowski JM, Brown DG, Neufeld JD. PANDAseq: pairedend assembler for illumina sequences. BMC Bioinformatics. 2012 Feb;13(1):31. 
17 De La Vega Elena CD, Nogués N, Fernández Montoya A, Chialina S, Blanzaco PD, Theiller E, et al. Human platelet-specific antigens frequencies in the Argentinean population. Transfus Med. 2008 Apr;18(2):83-90.

18 Lek M, Karczewski KJ, Minikel EV, Samocha KE, Banks E, Fennell T, et al.; Exome Aggregation Consortium. Analysis of protein-coding genetic variation in 60,706 humans. Nature. 2016 Aug;536(7616):285-91.

19 Peterson JA, Gitter ML, Pietz B, Bellissimo D, Curtis B, Aster R. A third allele of the HPA-5 $\mathrm{Br})$ platelet alloantigen system identified in investigating a case of neonatal thrombocytopenia. Transfusion. 2010 Aug;50(8):1855-6.

20 Santoso S, Kroll H, Andrei-Selmer CL, Socher I, Rankin A, Kretzschmar E, et al. A naturally occurring LeuVal mutation in $\beta 3$-integrin impairs the HPA-1a epitope: the third allele of HPA-1. Transfusion. 2006 May;46(5):790-9.

21 Bertrand G, Danger Y, Croisille L, Le Toriellec E, Verite F, Renac V, et al. A new platelet alloantigen (Efsa, HPA-35bw) on glycoprotein IIIa leading to neonatal alloimmune thrombocytopenia. Transfusion. 2019 Jul; 59(7):2463-4.

22 Bhatti FA, Uddin M, Ahmed A, Bugert P. Human platelet antigen polymorphisms (HPA$1,-2,-3,-4,-5$ and -15$)$ in major ethnic groups of Pakistan. Transfus Med. 2010 Apr;20(2): 78-87.

23 Neffati A, Sellami MH, Bellali H, Kaabi H, Chhabene M, Hmida S. Polymorphism of human platelet antigens in Tunisian popu- lation: clincal and anthropological interests. Transfus Clin Biol. 2019 Nov;26(4): 266-72.

24 Ikeda H, Mitani T, Ohnuma M, Haga H, Ohtzuka S, Kato T, et al. A new platelet-specific antigen, Naka, involved in the refractoriness of HLA-matched platelet transfusion. Vox Sang. 1989;57(3):213-7.

25 Carr R, Hutton JL, Jenkins JA, Lucas GF, Amphlett NW. Transfusion of ABO-mismatched platelets leads to early platelet refractoriness. Br J Haematol. 1990 Jul;75(3):408-13.

26 Balz V, Krause S, Fischer J, Enczmann J. More than 150 novel variants of HLA class I genes detected in German Stem Cell Donor Registry and UCLA International Cell Exchange samples. HLA. 2018 Mar;91(3):187-94. 